\title{
Proposed Cutoff Value of Brachial-Ankle Pulse Wave Velocity for the Management of Hypertension
}

Toshiaki Ohkuma, MD; Hirofumi Tomiyama, MD; Toshiharu Ninomiya, MD; Kazuomi Kario, MD; Satoshi Hoshide, MD; Yoshikuni Kita, PhD; Toyoshi Inoguchi, MD; Yasutaka Maeda, MD;

Katsuhiko Kohara, MD; Yasuharu Tabara, MD; Motoyuki Nakamura, MD; Takayoshi Ohkubo, MD; Hirotaka Watada, MD; Masanori Munakata, MD; Mitsuru Ohishi, MD; Norihisa Ito, MD;

Michinari Nakamura, MD; Tetsuo Shoji, MD; Charalambos Vlachopoulos, MD;

Akira Yamashina, MD on behalf of the Collaborative Group for Japan Brachial-Ankle pulse wave VELocity individual participant data meta-analysis of prospective studies (J-BAVEL)

Background: The optimal cutoff values of the brachial-ankle pulse wave velocity (baPWV) for predicting cardiovascular disease (CVD) were examined in patients with hypertension.

Methods and Results: A total of 7,656 participants were followed prospectively. The hazard ratio for the development of CVD increased significantly as the baPWV increased, independent of conventional risk factors. The receiver-operating characteristic curve analysis showed that the optimal cutoff values for predicting CVD was $18.3 \mathrm{~m} / \mathrm{s}$. This cutoff value significantly predicted THE incidence of CVD.

Conclusions: The present analysis suggests that the optimal cutoff value for CVD in patients with hypertension is $18.3 \mathrm{~m} / \mathrm{s}$.

Key Words: Arterial stiffness; Hypertension; Pulse wave velocity

$\mathbf{S}$ everal prospective studies have demonstrated that arterial stiffness, which is assessed by measuring the pulse wave velocity (PWV), is an independent risk factor for cardiovascular disease (CVD)., ${ }^{1,2}$ In the clinical setting, carotid-femoral PWV (cfPWV) and brachial-ankle PWV (baPWV) are available as measures of arterial stiffness. ${ }^{1}$ The 2013 ESH/ESC guidelines for the management of arterial hypertension described cfPWV $\geq 10 \mathrm{~m} / \mathrm{s}$ as a marker of vascular damage, and as an important parameter in deciding the most appropriate strategy for the manage- ment of hypertension. ${ }^{3}$ Because it is simple to measure (i.e., just by wrapping pressure cuffs around the upper arms and lower legs), baPWV measurement is more widely applicable. ${ }^{1}$ Recently, we reported from an individual participant data (IPD) meta-analysis that the baPWV can enhance the efficacy of risk prediction of development of CVD over that of the Framingham risk score. ${ }^{2}$ We conducted the present study to determine the cutoff value of baPWV for predicting the development of CVD (i.e., as a marker of vascular damage) in subjects with hypertension.

Received July 3, 2017; revised manuscript received July 26, 2017; accepted August 1, 2017; released online August 24,2017 Time for primary review: 20 days

Department of Medicine and Clinical Science, Graduate School of Medical Sciences (T. Ohkuma), Department of Epidemiology and Public Health, Graduate School of Medical Sciences (T.N.), Innovation Center for Medical Redox Navigation (T.I.), Department of Medicine and Bioregulatory Science, Graduate School of Medical Sciences (Y.M.), Kyushu University, Fukuoka; Department of Cardiology, Tokyo Medical University, Tokyo (H.T., A.Y.); Division of Cardiovascular Medicine, Department of Medicine, Jichi Medical University School of Medicine, Tochigi (K. Kario, S.H.); Faculty of Nursing Science, Tsuruga Nursing University, Fukui (Y.K.); Department of Regional Resource Management, Faculty of Collaborative Regional Innovation, Ehime University, Ehime (K. Kohara); Center for Genomic Medicine, Kyoto University Graduate School of Medicine, Kyoto (Y.T.); Department of Internal Medicine, Iwate Medical University, Iwate (Motoyuki N.); Department of Hygiene and Public Health, Teikyo University School of Medicine, Tokyo (T. Ohkubo); Departments of Metabolism \& Endocrinology, Juntendo University, Graduate School of Medicine, Tokyo (H.W.); Research Center for Lifestyle-related Disease, Tohoku Rosai Hospital, Miyagi (M.M.); Department of Cardiovascular Medicine and Hypertension, Graduate School of Medical and Dental Sciences, Kagoshima University, Kagoshima (M.O.); Department of Geriatric and General Medicine, Osaka University Graduate School of Medicine, Osaka (N.I.); Cardiovascular Institute Japan, Tokyo (Michinari N.); Department of Vascular Medicine, Osaka City University Graduate School of Medicine, Osaka (T.S.), Japan; and Hypertension and Cardiometabolic Unit, (1st) Department of Cardiology, Athens Medical School, Hippokration Hospital, Athens (C.V.), Greece

Collaborators are listed in the Appendix S1.

Mailing address: Hirofumi Tomiyama, MD, FAHA, Department of Cardiology, Tokyo Medical University, 6-7-1 Nishi-Shinjuku-ku, Tokyo 160-0023, Japan. E-mail: tomiyama@tokyo-med.ac.jp

ISSN-1346-9843 All rights are reserved to the Japanese Circulation Society. For permissions, please e-mail: cj@j-circ.or.jp 

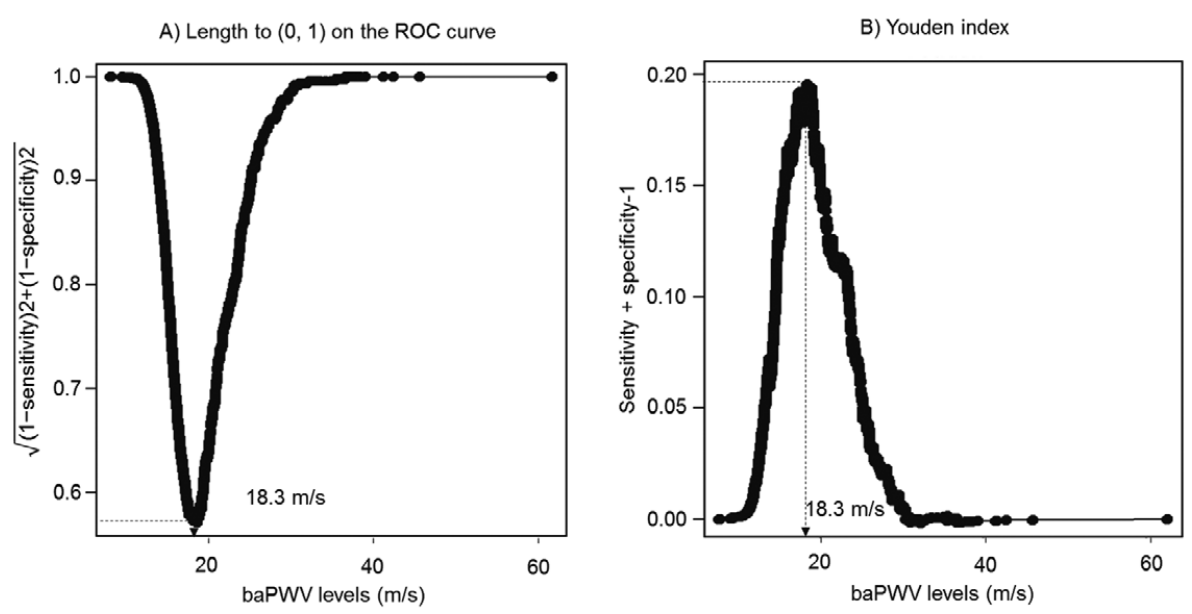

Figure. Cutoff value of brachial-ankle pulse wave velocity (baPWV) that allows for optimal discrimination between patients at risk and not at risk of development of cardiovascular disease in the future: $(\mathbf{A})$ the length to $(0,1)$ on the receiver-operating characteristics (ROC curve) and (B) the Youden Index.

\begin{tabular}{|c|c|c|c|c|c|}
\hline \multirow{2}{*}{ baPWV (m/s) } & \multirow{2}{*}{$\begin{array}{l}\text { No. of events/ } \\
\text { no. of subjects }\end{array}$} & \multicolumn{2}{|c|}{ Age- and sex-adjusted } & \multicolumn{2}{|c|}{ Multivariate-adjusted $^{*}$} \\
\hline & & HR (95\% Cl) & $P$ value & HR (95\% Cl) & $P$ value \\
\hline \multicolumn{6}{|c|}{ CVD $(n=7,656$, events $=486)$} \\
\hline$<18.30$ & $213 / 4,756$ & 1.00 (Ref.) & & 1.00 (Ref.) & \\
\hline$\geq 18.30$ & $273 / 2,900$ & $1.33(1.09-1.63)$ & $<0.001$ & $1.33(1.06-1.67)$ & 0.01 \\
\hline
\end{tabular}

The Ehime Study, Hisayama Study, Iwate Study, Ohasama Study, Takashima Study, the Japan Morning Surge Home Blood Pressure (J-HOP) study, the Non-invasive Atherosclerotic evaluation of Hypertension (NOAH) study, and Kyushu Prevention Study of Atherosclerosis study cohorts were included in the analysis. CVD in the Ohasama study was defined as a composite of CVD death and stroke. *Adjusted for age, sex, brachial systolic blood pressure, history of use of antihypertensive agents, hemoglobin A1c, body mass index, serum total cholesterol, serum high-density lipoprotein cholesterol, and current smoking habit. Subjects without missing values for covariates were included in the multivariate-adjusted model $(n=6,933)$. baPWV, brachial-ankle pulse wave velocity; $\mathrm{Cl}$, confidence interval; CVD, cardiovascular disease; HR, hazard ratio.

\section{Methods}

Japan Brachial-Ankle pulse wave VELocity individual participant data meta-analysis of prospective studies (J-BAVEL) is an IPD meta-analysis examining the association of baPWV with the risk of development of CVD. Previous IPD meta-analysis ${ }^{2}$ included 14,673 participants without pre-existing CVD from 8 studies (5 published studies $^{\mathbf{4} 8}$ and 3 unpublished studies $\left.{ }^{9-11}\right)$. Among them, 7,656 subjects with hypertension (defined as blood pressure (BP) $\geq 140 / 90 \mathrm{mmHg}$ and/or current use of antihypertensive agents) were included in the present study. During the follow-up period, the patients' vital status and incidence of CVD (defined as the incidence of stroke and ischemic heart disease) were ascertained.

The pooled hazard ratio (HR) and 95\% confidence intervals (CIs) for the outcomes were estimated using a stratified Cox proportional hazards regression model, in which the cohort effect was adjusted as a fixed effect by taking each cohort as a strata variable. The pooled risk estimates per every 1 standard deviation (SD) increase of the baPWV levels were estimated by the relevant Cox model, with the baPWV included as a continuous variable. To confirm the optimal cutoff value of baPWV for predicting the future risk of CVD, receiver-operating characteristic (ROC) curves were plotted. The point on the ROC curve that yielded the figure closest to the ideal of $100 \%$ sensitivity and $100 \%$ specificity and the value that minimized the Youden Index were calculated.

\section{Results}

The mean age of the study subjects was 64 years, and the proportion of men was $47 \%$. Mean values of baPWV, brachial systolic BP, brachial diastolic BP, hemoglobin Alc $\left(\mathrm{HbA}_{1 \mathrm{c}}\right)$, body mass index (BMI), serum total cholesterol, serum high-density lipoprotein (HDL) cholesterol, and estimated glomerular filtration rate ${ }^{\mathbf{1 2}}$ were $17.87 \mathrm{~m} / \mathrm{s}$, $146 \mathrm{mmHg}, 85 \mathrm{mmHg}, 6.1 \%, 24.3 \mathrm{~kg} / \mathrm{m}^{2}, 206.5 \mathrm{mg} / \mathrm{dL}$, $58.5 \mathrm{mg} / \mathrm{dL}$, and $72.8 \mathrm{~mL} / \mathrm{min} / 1.73 \mathrm{~m}^{2}$, respectively. The proportion of subjects with a current smoking habit was $17 \%$.

During the follow-up period, a total of 486 participants developed CVD. The age- and sex-adjusted pooled HR for the development of CVD increased by $14 \%$ (95\% CI, $5-25 \%$ ) per every 1 SD increase of the baPWV. This association remained significant even after adjusting for age, sex, brachial systolic BP, history of use of antihypertensive 
agents, $\mathrm{HbA}_{1 \mathrm{c}}, \mathrm{BMI}$, serum total cholesterol, serum HDL cholesterol, and current smoking habit (HR, 1.15; 95\% CI 1.04-1.27).

The optimal cutoff point of baPWV on the ROC curve that yielded a value closest to the ideal of $100 \%$ sensitivity and $100 \%$ specificity, and the point that minimized the Youden Index was baPWV $=18.3 \mathrm{~m} / \mathrm{s}$ (Figure). The sensitivity and specificity of this cutoff value were $56.2 \%$ and $63.4 \%$, respectively, and the area under the ROC curve was 0.629 . This cutoff value significantly predicted incidence of CVD after adjustment for the aforementioned confounding factors (Table; multivariate-adjusted HR, 1.33; 95\% CI 1.06-1.67).

\section{Discussion}

In the guidelines for the management of hypertension, ${ }^{3,13}$ the assessment of subclinical organ damage is an important clue to determining the most appropriate strategy for the management of hypertension. The IPD meta-analysis demonstrated that the cfPWV is an independent risk factor for the future development of CVD, ${ }^{14}$ and the $2013 \mathrm{ESC} /$ ESH guideline for the management of hypertension proposed cfPWV as a marker of subclinical organ damage. ${ }^{3}$ As previously reported, according to an analysis using a multivariate model with adjustment for the conventional risk factors for CVD, baPWV is an independent risk factor for the development of CVD, but not for all-cause death. ${ }^{2}$ The present study identified baPWV $\geq 18.3 \mathrm{~m} / \mathrm{s}$ as a cutoff value for the development of CVD in subjects with hypertension.

Increased arterial stiffness is thought to increase the risk of development of CVD via a variety of mechanisms other than atherosclerotic plaque formation. ${ }^{2}$ However, subjects with hypertension are likely to have endothelial dysfunction and vascular inflammation, potentially leading to plaque formation and rupture. ${ }^{\mathbf{1} 2}$ Our analysis revealed that the optimal cutoff value derived from the ROC curve was a significant predictor for the development of CVD, independent of conventional risk factors. Therefore, it may be reasonable to apply baPWV $\geq 18.3 \mathrm{~m} / \mathrm{s}$ as a marker of subclinical organ damage in the CVD risk assessment of patients with hypertension. Although we should make clear that the use of a threshold is meant to simplify the estimation of risk by physicians in everyday clinical practice, this cutoff value is not the cutoff point for binary discrimination of the patient's cardiovascular risk status, because the cardiovascular risk increases continuously along with the increase of the baPWV values (e.g., the cut-of value in subjects including the general population is $15.9 \mathrm{~m} / \mathrm{s}){ }^{2}$

In conclusion, in subjects with hypertension, the baPWV is a predictor of the risk of development of CVD, independent of the conventional risk factors, and $18.3 \mathrm{~m} / \mathrm{s}$ may be considered as a reference value for the cardiovascular risk assessment.

\section{Sources of Funding}

This study was supported by Omron Health Care Company (Kyoto, Japan), which awarded a grant to the baPWV IPD meta-analysis study group.

\section{Disclosures}

H.T. and A.Y. received research grants from Omron Health Care Company (Kyoto, Japan) and Asahi food and health care (Tokyo, Japan).

\section{References}

1. Tomiyama H, Matsumoto C, Shiina K, Yamashina A. Brachialankle PWV: Current status and future directions as a useful marker in the management of cardiovascular disease and/or cardiovascular risk factors. J Atheroscler Thromb 2016; 23: $128-146$.

2. Ohkuma T, Ninomiya T, Tomiyama H, Kario K, Hoshide S, Kita Y, et al; Collaborative Group for J-BAVEL (Japan BrachialAnkle Pulse Wave Velocity Individual Participant Data MetaAnalysis of Prospective Studies). Brachial-Ankle Pulse Wave Velocity And The Risk Prediction Of Cardiovascular Disease: An Individual Participant Data Meta-Analysis. Hypertension 2017; 69: 1045-1052.

3. Mancia G, Fagard R, Narkiewicz K, Redon J, Zanchetti A, Böhm M, et al. $2013 \mathrm{ESH} / \mathrm{ESC}$ guidelines for the management of arterial hypertension: The Task Force for the Management of Arterial Hypertension of the European Society of Hypertension (ESH) and of the European Society of Cardiology (ESC). Eur Heart J 2013; 34: 2159-2219.

4. Ninomiya T, Kojima I, Doi Y, Fukuhara M, Hirakawa Y, Hata $\mathrm{J}$, et al. Brachial-ankle pulse wave velocity predicts the development of cardiovascular disease in a general Japanese population: The Hisayama study. J Hypertens 2013; 31: 477-483.

5. Ishisone T, Koeda Y, Tanaka F, Sato K, Nagano M, Nakamura M. Comparison of utility of arterial stiffness parameters for predicting cardiovascular events in the general population. Int Heart $J$ 2013; 54: 160-165.

6. Turin TC, Kita Y, Rumana N, Takashima N, Kadota A, Matsui $\mathrm{K}$, et al. Brachial-ankle pulse wave velocity predicts all-cause mortality in the general population: Findings from the Takashima study, Japan. Hypertens Res 2010; 33: 922-925.

7. Kawai T, Ohishi M, Onishi M, Ito N, Takeya Y, Maekawa Y, et al. Cut-off value of brachial-ankle pulse wave velocity to predict cardiovascular disease in hypertensive patients: A cohort study. J Atheroscler Thromb 2013; 20: 391-400.

8. Maeda Y, Inoguchi T, Etoh E, Kodama Y, Sasaki S, Sonoda N, et al. Brachial-ankle pulse wave velocity predicts all-cause mortality and cardiovascular events in patients with diabetes: The Kyushu Prevention Study of Atherosclerosis. Diabetes Care 2014; 37: 2383-2390.

9. Kohara K, Ochi M, Okada Y, Yamashita T, Ohara M, Kato T, et al. Clinical characteristics of high plasma adiponectin and high plasma leptin as risk factors for arterial stiffness and related endorgan damage. Atherosclerosis 2014; 235: 424-429.

10. Hatanaka $\mathrm{R}$, Obara $\mathrm{T}$, Watabe $\mathrm{D}$, Ishikawa $\mathrm{T}$, Kondo $\mathrm{T}$, Ishikura $\mathrm{K}$, et al. Association of arterial stiffness with silent cerebrovascular lesions: The Ohasama study. Cerebrovasc Dis 2011; 31: 329-337.

11. Hoshide S, Yano Y, Haimoto H, Yamagiwa K, Uchiba K, Nagasaka S, et al. Morning and evening home blood pressure and risks of incident stroke and coronary artery disease in the Japanese general practice population: The Japan morning surgehome blood pressure study. Hypertension 2016; 68: 54-61.

12. Horio M, Imai E, Yasuda Y, Watanabe T, Matsuo S. Modification of the CKD epidemiology collaboration (CKD-EPI) equation for Japanese: Accuracy and use for population estimates. Am J Kidney Dis 2010; 56: 32-38.

13. Shimamoto K, Ando K, Fujita T, Hasebe N, Higaki J, Horiuchi M, et al; Japanese Society of Hypertension Committee for Guidelines for the Management of Hypertension. The Japanese Society of Hypertension Guidelines for the Management of Hypertension (JSH2014). Hypertens Res 2014; 37: 253-390.

14. Ben-Shlomo Y, Spears M, Boustred C, May M, Anderson SG, Benjamin EJ, et al. Aortic pulse wave velocity improves cardiovascular event prediction: An individual participant meta-analysis of prospective observational data from 17,635 subjects. $J$ Am Coll Cardiol 2014; 63: 636-646.

\section{Supplementary File 1}

\section{Supplementary Files}

Appendix S1. J-BAVEL Collaborators

Please find supplementary file(s);

http://dx.doi.org/10.1253/circj.CJ-17-0636 